\title{
Abnormal Fear Conditioning and Amygdala Processing in an Animal Model of Autism
}

\author{
Kamila Markram*,', Tania Rinaldi', Deborah La Mendola', Carmen Sandi' and Henry Markram' \\ 'Brain Mind Institute, Swiss Federal Institute of Technology (EPFL), Lausanne, Switzerland
}

\begin{abstract}
A core feature of autism spectrum disorders is the impairment in social interactions. Among other brain regions, a deficit in amygdala processing has been suggested to underlie this impairment, but whether the amygdala is processing fear abnormally in autism, is yet not clear. We used the valproic acid (VPA) rat model of autism to (a) screen for autism-like symptoms in rats, (b) test for alterations in amygdala-dependent fear processing, and (c) evaluate neuronal reactivity and synaptic plasticity in the lateral amygdala by means of in vitro single-cell electrophysiological recordings. VPA-treated animals displayed several symptoms common to autism, among them impaired social interactions and increased repetitive behaviors. Furthermore, VPA-treated rats were more anxious and exhibited abnormally high and longer lasting fear memories, which were overgeneralized and harder to extinguish. On the cellular level, the amygdala was hyperreactive to electrical stimulation and displayed boosted synaptic plasticity as well as a deficit in inhibition. We show for the first time enhanced, overgeneralized and resistant conditioned fear memories in an animal model of autism. Such hyperfear could be caused by the hyperreactivity and hyperplasticity found in the lateral amygdala, which may in turn be due to a deficit in the inhibitory system of the amygdala. We hypothesize an 'aversive world' syndrome that could, even if not a primary cause of the disorder itself, underlie some core symptoms in autism, such as impairments in social interactions and resistance to rehabilitation.

Neuropsychopharmacology (2008) 33, 90 I-912; doi:10.1038/sj.npp. I 301453; published online 16 May 2007
\end{abstract}

Keywords: amygdala; autism; fear memory; fear extinction; synaptic plasticity; valproic acid

\section{INTRODUCTION}

Autism is one of the fastest growing developmental disorders characterized by inhibited reciprocal social interactions, communication deficits and marked inflexibility to environmental changes, which makes rehabilitation exceedingly difficult. Several brain structures and pathways have been suggested to underlie this disorder, among them the amygdala (Bachevalier, 1994; Baron-Cohen et al, 1999; Adolphs et al, 2001), which exhibits a number of abnormalities. Whereas earlier postmortem studies on autistic patients indicated an increased number of cells with reduced cell size in the cortical, medial, and central nuclei of the amygdala (Kemper and Bauman, 1998), more recent and modern stereological counts of neurons in the amygdala revealed a reduced number of neurons, particularly in the lateral nucleus (Schumann and Amaral, 2006). The total amygdala volume is enlarged during early infancy (Sparks et al, 2002; Schumann et al, 2004), but can be reduced in adulthood (Aylward et al, 1999). Functionally,

\footnotetext{
*Correspondence: Dr $\mathrm{K}$ Markram, Brain Mind Institute, Swiss Federal Institute of Technology (EPFL), Building AAB 102, Station 15, I0I5 Lausanne, Switzerland, Tel: +4I 216931642 ,

E-mail: kamila.markram@epfl.ch

Received II December 2006; revised 10 April 2007; accepted I 3 April 2007
}

the amygdala has been linked to autism through its involvement in socio-emotional behavior. Neuroimaging studies indicate that the amygdala does not activate sufficiently when subjects view faces or judge feelings and thoughts from faces (Baron-Cohen et al, 1999; Critchley et al, 2000; Pierce et al, 2001), which may indicate weak amygdala functioning in autism. Monkey studies seem to support this hypothesis as amygdala-lesioned monkeys withdraw socially, fail to initiate and respond to social behaviors (Kling and Brothers, 1992), and exhibit flat vocalizations that lack affect (Newman and Bachevalier, 1997). However, the hypothesis that the amygdala plays a fundamental role in the generation of social behavior has been questioned more recently (Amaral et al, 2003) and there might be an alternative view on the involvement of the amygdala in autism. Kanner's original (Kanner, 1943) and other more recent studies (Muris et al, 1998; Gillott et al, 2001; Evans et al, 2005) suggest that abnormal anxiety, fears and phobias are also traits of autism, and the amygdala has been extensively linked to both anxiety and conditioned fear, in humans and in animals (Davis and Whalen, 2001; LeDoux, 2003). Thus, it is possible that abnormal processing in the amygdala might be central to the generation of these excessive fears observed in autism.

To test this hypothesis, we assessed fear processing in the valproic acid (VPA) rat model of autism (Rodier et al, 1996). VPA is one of the teratogens implicated in causing 
autism in humans (Christianson et al, 1994; Williams and Hersh, 1997; Moore et al, 2000; Williams et al, 2001; Rasalam et al, 2005), when administered around the critical time period of neural tube closure (embryonic day 20-24; Arndt et al, 2005). In rats, a single intraperitoneal (i.p.) injection of VPA to the pregnant dam at a corresponding time of embryonic day 12.5 tends to produce a neuroanatomical and behavioral phenotype similar to the human condition. Offspring of treated dams exhibit brain stem injuries, diminished number of Purkinje cells in the cerebellum, impaired social interaction, increased repetitive behavior, increased nociceptive thresholds, increased sensitivity to complex sensory stimulation, and enhanced anxiety (Rodier et al, 1996, 1997; Ingram et al, 2000; Schneider and Przewlocki, 2005; Schneider et al, 2006), all symptoms that are common to autism.

The present study had three approaches: first, animals were screened for autism-like symptoms to further validate the VPA rat model of autism. Second, amygdala function was evaluated on the behavioral level by assessing fear memories and fear processing. Finally, the amygdala was evaluated on the electrophysiological level to check for possible alterations in neuronal processing and synaptic plasticity.

The results were previously presented in abstract and poster form at Society for Neuroscience and Federation of European Neurosciences conferences (Markram et al, 2005; La Mendola et al, 2006).

\section{MATERIALS AND METHODS}

\section{Valproic Acid Model of Autism}

Wistar Han rats (Charles River Laboratories, L'Arbresle, France) were mated and pregnancy was determined by the presence of a vaginal plug on embryonic day 1 (E1). Sodium salt of valproic acid (NaVPA, Sigma) was dissolved in $0.9 \%$ saline for a concentration of $150 \mathrm{mg} / \mathrm{ml}$ ( $\mathrm{pH} 7.3)$. The dosing volume was $3.3 \mathrm{ml} / \mathrm{kg}$; the dosage was adjusted according to the body weight of the dam on the day of injection. Treated dams received a single ip injection of $500 \mathrm{mg} / \mathrm{kg} \mathrm{NaVPA}$ and control dams a single ip injection of saline on E12.5. Dams were housed individually and were allowed to raise their own litters until weaning (postnatal day P23). The offspring was then separated and housed in cages of 3-4 rats until the start of behavioral experiments (approximately 3 months after birth). Electrophysiological experiments were conducted on P12-P16. Overall, for behavioral procedures, 274 male and female rat offspring (136 controls, 138 VPAtreated) from 36 litters (16 control, 20 VPA-treated) were tested. In electrophysiological experiments, 13 male rat offspring (six controls, seven VPA-treated) from 11 litters (six control, five VPA-treated) were tested. The birth rate was approximately $25 \%$ lower in VPA- than saline-injected dams, which was reflected in lower weight of the dams during pregnancy. Whereas there were no differences in weight before the injection (saline: $277.7 \mathrm{~g} \pm 4.0$; VPA: $283.9 \mathrm{~g} \pm 2.4 ; t_{14}=-1.40, p=0.18$ ), saline-injected dams gained significantly more weight 5 days after the injection than VPA-injected dams (saline: $50.2 \mathrm{~g} \pm 3.1$; VPA: $\left.15.7 \mathrm{~g} \pm 2.5 ; t_{14}=8.84, p<0.000\right)$.
All the procedures described were conducted in conformity with the Swiss National Institutional Guidelines on Animal Experimentation on the ethical use of animals, and approved by the Swiss Cantonal Veterinary Office Committee for Animal Experimentation.

\section{Social Interaction}

Rats were separated and housed individually the night before the experiment to enhance later social interactions. The apparatus was a white plastic box $(50 \times 40 \times 40 \mathrm{~cm})$ containing a plastic tube big enough for a rat to hide inside. Rats were matched for their gender and weight. Pairs of either treated or control rats were put into the apparatus over a period of $20 \mathrm{~min}$. The percent time spent pinning (one rat lies on its back, the other stands with two paws on top of it), following, touching, grooming each other, sniffing of any body part besides of anogenital parts, sniffing of the anogenital body parts and hiding inside the tube during the first and second $10 \mathrm{~min}$ were taken as indicators of social engagement.

\section{Repetitive Behavior}

Rats were tested in the spontaneous alteration paradigm. Rats were inserted individually into the start arm of a standard Y-maze (gray polyvinyl plastic) and allowed to move freely on each of two trials. On trial 1, the animal was allowed to enter either arm, neither of which was rewarded, and remain there for $5 \mathrm{~s}$. The rat was then returned to the start arm and the subsequent arm choice was recorded (trial 2). The percentage of rats per group re-entering the same arm was taken as an indicator for repetitive behavior.

\section{Nociception}

Thermal pain sensitivity was measured with the hot plate test (TSE, Bad Homburg, Germany). The plate was set to a temperature of $50^{\circ} \mathrm{C}$ and the latency to a discomfort reaction (paw withdrawal) was measured. Cut-off time was $1 \mathrm{~min}$.

\section{Startle Response and Prepulse Inhibition}

Acoustic startle reflex amplitude and prepulse inhibition (PPI) were measured with the SR-Lab System (San Diego Instruments, CA, USA). The equipment included response platforms that were individually calibrated and placed in sound attenuating chambers. Each chamber was equipped with an internal light, fan, and sound generation system. Plexiglas cylinders large enough to hold adult rats with minimal restraint were mounted on the platforms. The white background noise was adjusted to a constant $70 \mathrm{~dB}$.

The test session was conducted in three blocks and started with a 5 min acclimation period. Block 1 consisted of five startle-only trials with a $115 \mathrm{~dB}$ acoustic stimulus pulse (startle tone). Block 2 had a total of 36 trials: 12 startle-only trials as in block 1 and 24 prepulse plus startle trials, conducted under four different conditions: the intensity of the prepulse was either 78 or $86 \mathrm{~dB}$ and the prepulse to startle tone interval was either 30 or $120 \mathrm{~ms}$. The trials were presented in random order with the intertrial duration 
ranging from 10 to $20 \mathrm{~s}$. Block 3 had an additional five startle-only trials. Each stimulus had a $2 \mathrm{~ms}$ rise/fall time. The entire test period lasted approximately $25 \mathrm{~min}$.

\section{Anxiety}

Rats were inserted for $5 \mathrm{~min}$ in a standard elevated plusmaze, which consisted of two opposite open arms and two opposite closed arms $(49 \times 10 \times 42 \mathrm{~cm})$ arranged at right angles. The percent time spent in the open and closed arms indicated anxiety levels and the total distance $(\mathrm{cm})$ moved and velocity $(\mathrm{cm} / \mathrm{s})$, with which rats moved through the entire maze, indicated locomotor and exploration capabilities.

\section{Fear Conditioning}

Fear conditioning occurred in a standard fear conditioning apparatus (Panlab, Barcelona, Spain). The floor consisted of 20 steel rods through which a scrambled shock could be delivered. The sidewalls of the observation cage were of black stainless steel and the door of Plexiglas. Auditory fear conditioning (AFC) training started with a $160 \mathrm{~s}$ habituation period, followed by three $1 \mathrm{~mA}$ shocks (inter-shock interval: $60 \mathrm{~s})$, each paired with a $20 \mathrm{~s}$ tone $(800 \mathrm{~Hz}, 80 \mathrm{~dB})$. Ethanol (4\%) was used for cleaning in between training sessions. Fear memories to the context were assessed by putting the rats for $8 \mathrm{~min}$ into exactly the same context as during training, but without shocks. To measure fear memories to the tone, visual (green plastic walls) and odor (4\% clorine) cues in the conditioning chamber were exchanged and rats remained in the chamber for $8 \mathrm{~min}$, during which the tone was continuously on for the last $5 \mathrm{~min}$. The percent time spent freezing during the $8 \mathrm{~min}$ context and $5 \mathrm{~min}$ tone memory test were taken as indicators of fear. Fear memories to the context and tone were assessed on two successive days, starting 1 day, 30 and 90 days post-training.

Fear generalization to a novel context and tone was performed on day 4 and assessed as in the tone memory test, just that the tone was additionally changed as well $(400 \mathrm{~Hz}, 80 \mathrm{~dB})$. Freezing during the first $3 \mathrm{~min}$ indicated fear generalization to a novel context and during the last $5 \mathrm{~min}$ to a novel tone.

To assess fear extinction, the same animals that were previously evaluated for fear memories were divided into three groups and were first re-conditioned (three shocks, $0.5 \mathrm{~mA}$ ) either to the context alone (contextual fear conditioning (CFC)) or paired with a tone (as above) 1 day after the last memory test. Fear extinction was then conducted under three conditions: the group that received CFC was extinguished to the same context on four subsequent days, starting with an $8 \mathrm{~min}$ pre-extinction context test, followed by two days of $30 \mathrm{~min}$ further context exposure and ending with another $8 \mathrm{~min}$ post-extinction context test. Rats, which received AFC, were extinguished in either the same or a different context as during training. The procedure was as above: first a 5 min pre-extinction tone memory test was conduced, followed by two extinction sessions (each $30 \mathrm{~min}$ in which a $20 \mathrm{~s}$ tone alternated with $40 \mathrm{~s}$ silence) and finally a $5 \mathrm{~min}$ post-extinction tone test. For each animal in all conditions, an extinction index was computed describing the percentual decrease in freezing from pre-extinction (=baseline) to post-extinction test (freezing post-extinction/freezing pre-extinction $\times 100$ ).

\section{Morris Water Maze}

The water maze apparatus consisted of a large circular pool (2.05 $\mathrm{m}$ in diameter) filled with water $\left(25 \pm 1^{\circ} \mathrm{C}\right)$. A platform $(11 \mathrm{~cm}$ diameter) was submerged $1 \mathrm{~cm}$ under the water surface. Both pool and platform were made of black polyvinyl plastic and offered no intramaze cues to guide escape behavior. The water maze was surrounded with curtains containing several extramaze visual cues.

Rats received 4 days of spatial training, each session consisting of four trials (intertrial interval (ITI): $30 \mathrm{~s}$ ). Each trial started with the rat facing the wall at one of four possible positions. The latency to find the platform was measured. If a rat did not find the platform within $90 \mathrm{~s}$, it was guided towards it. Each rat remained on the platform for $15 \mathrm{~s}$ before it would be taken out. On day 5 , a probe test was conducted, in which the platform was removed from the pool and animals were released to the pool for a 60-s period from the quadrant opposite to the one where the platform had been previously located. At the end of the probe trial the platform was re-inserted into the pool and rats remained on it for $15 \mathrm{~s}$.

Video tracking software (Ethovision, Noldus, The Netherlands) was used for automatic recording and analysis of escape latencies, distances swum and velocities in case of the spatial training sessions and percentage of time spent and distance swum in the target quadrant, number of virtual platform crossings, and latency to reach the virtual platform in case of the probe trial.

\section{Acute Slice Preparation for In Vitro Electrophysiology}

Rats (P12-P16) were rapidly decapitated and coronal amygdala slices $(300 \mu \mathrm{m}$ thick $)$ were sectioned on a vibratome (HR2, Sigmann Elektronik) in iced artificial cerebral spinal fluid (ACSF, contained (mM): $125 \mathrm{NaCl}, 2.5$ $\mathrm{KCl}, 25$ glucose, $25 \mathrm{NaHCO}_{3}, 1.25 \mathrm{NaH}_{2} \mathrm{PO}_{4}, 2 \mathrm{CaCl}_{2}$ and 1 $\mathrm{MgCl}_{2}$ ). Slices were incubated for $30 \mathrm{~min}$ at $35^{\circ} \mathrm{C}$ and then at room temperature until transferred to the recording chamber (room temperature or $34^{\circ} \mathrm{C}$ ). Neurons in the lateral amygdaloid nucleus (LA) were identified using IRDIC microscopy, with an upright microscope (Olympus BX51WI, fitted with a $60 \times / 0.90 \mathrm{~W}$ objective, Olympus, Switzerland). Recorded neurons were selected up to $70 \mu \mathrm{m}$ below the slice surface.

\section{Electrophysiological Recording}

Simultaneous whole-cell recordings from clusters of up to four pyramidal neurons (pipette resistance 4-10 M $\Omega$ ) were made in the LA and signals were amplified using Axopatch 200B amplifiers (Axon Instruments, CA, USA). Voltages (in current-clamp mode) or currents (in voltage-clamp mode) were recorded with pipettes containing (in $\mathrm{mM}$ ): 110 potassium gluconate, $10 \mathrm{KCl}, 4 \mathrm{ATP}-\mathrm{Mg}, 10$ phosphocreatine, $0.3 \mathrm{GTP}, 10$ Hepes, and $0.5 \%$ biocytin ( $\mathrm{pH} 7.3$, 270-300 mOsm). Membrane potentials were not corrected for the junction potentials between pipette and bath solution $(\sim 10 \mathrm{mV})$. 


\section{Multi-Electrode Array Stimulation}

Mea stimulation. Multisite extracellular stimulations were performed using a multi-electrode array (MEA) made of 60 3D platinum electrodes (Ayanda Biosystems, EPFL, Switzerland), on top of which acute brain slices were glued with a nitrocellulose solution $(0.14 \mathrm{mg} / \mathrm{ml}$ in ethanol). Responses to these MEA stimulations were recorded in whole-cell patched pyramidal cells in LA. To study the effects of network stimulation, 16 MEA electrodes were stimulated simultaneously with a Poisson train $(50 \mathrm{~Hz}, 300 \mathrm{~ms})$ at increasing stimulation amplitude $(0.1-2 \mathrm{~V})$. Whole-cell voltage-clamped cells were then recorded at $-80 \mathrm{mV}$ (reversal potential of GABA receptors) to study excitatory effects and at $+10 \mathrm{mV}$ (reversal potential of AMPA receptors) for the isolation of inhibitory inputs.

\section{Long-Term Potentiation}

An extracellular electrode was placed $100-300 \mu \mathrm{m}$ medial to the LA in the ventral striatum (Bauer et al, 2002). Wholecell patched pyramidal cells were recorded in the LA. Calibration procedures for long-term potentiation (LTP) recordings were conducted according to Bauer et al (2002). A stimulation pulse of an amplitude producing a response of a few millivolts in the I-clamped patched cell was given before the pairing protocol, and the maximum amplitude of the mean of 30 traces was measured. The $3 \mathrm{~s}$ long pairing protocol consisted of a $30 \mathrm{~Hz}$ regular train stimulation to the extracellular electrode simultaneously with a depolarization above threshold of the patched cell, applied three times at $30 \mathrm{~s}$ intervals. The response to the stimulation pulse was then monitored up to $30 \mathrm{~min}$ after the initial recording (three sets of 30 stimulation pulses every $10 \mathrm{~s}$, with $5 \mathrm{~min}$ interval between each set). The percent increase in the response amplitude to the stimulation pulse after pairing compared to before pairing was measured.

\section{Statistical Analysis}

For comparison of rates and means, two-sided $\chi^{2}$ and Student's $t$-tests were used, respectively. ANOVA for repeated measurements was applied when sequences were evaluated (social interaction, spatial training sessions on each of the 4 days, network inhibition at increasing stimulation amplitudes). Post-hoc analysis was performed with Student's $t$-tests. Statistics reported in the text and figures represent the mean \pm SEM.

\section{RESULTS}

\section{Behavioral Validation of the VPA Model of Autism}

Before exploring fear processing in the VPA rat model, we first wanted to test whether the behavioral deficits previously reported (Schneider and Przewlocki, 2005), were reproduced in our animals.

Impaired social interaction. For social interaction, we evaluated how pairs of unfamiliar VPA-treated or control animals interacted freely for $20 \mathrm{~min}$ in a box containing an escape tube large enough for a rat. Treated animals exhibited less play behavior (pinning, $\mathrm{F}_{1,18}=8.14$, $p=0.01$ ), explored each other less, as indicated by sniffing $\left(\mathrm{F}_{1,18}=23.21, p<0.000\right)$ and touching each other $\left(\mathrm{F}_{1,18}=4.25, p=0.05\right)$, and avoided interaction by hiding more in the tube than control animals $\left(\mathrm{F}_{1,18}=4.25\right.$, $p=0.05)$, but did not differ from control animals in their grooming $\left(\mathrm{F}_{1,18}=1.94, p=0.2\right)$ and anogenital inspections $\left(\mathrm{F}_{1,18}=0.51, p=0.58 ; n=10\right.$ pairs/group; Figure 1a) of each other. Therefore, VPA-treated rats show deficits in some, but not all of the social interaction behaviors performed by rats.

Increased repetitive behavior. Repetitive behavior was tested in a spontaneous alternation task in the Y-maze (Kahne et al, 2002), which is thought to reveal obsessivecompulsive-like behaviors (Yadin et al, 1991). Once the rat entered and briefly explored one of the two possible arms in the first trial, it was re-put into the starting position for the second trial and the entrance into either a novel or the same arm previously entered was monitored. Normally, rats prefer to explore a novel arm in the second trial. In accordance with this, only $24 \%$ of the control rats $(n=43)$ re-entered the same arm. However, $51 \%$ of treated rats $(n=35)$ re-entered the same arm $\left(\chi_{1}^{2}=6.05, p=0.01\right.$; Figure $1 b$ ), suggestive of repetitive tendencies.

Impaired thermal nociception. VPA-treated offspring $(n=39)$ exhibited highly significantly higher latencies to withdraw the hindpaw in the hot plate test than controls $(n=36)\left(t_{73}=4.62 ; p=0.000\right)$, indicating impaired sensitivity to thermal pain.

Intact sensory processing, but impaired sensorimotor gating. As increased sensitivity to sensory stimulation is also symptomatic of autism, we measured the startle response to a tone alone ( $115 \mathrm{~dB}$, the startle tone). Further, we examined PPI, that is, when the tone was preceded by another less-intense tone (a prepulse tone). In this configuration the softer prepulse inhibits the response to the second louder tone and the response is smaller than when the sound is presented on its own, which may be interpreted as sensory habituation and/or response inhibition. There was no difference in response to the startle tone between control $(n=58)$ and treated $(n=56)$ animals $\left(t_{112}=0.01, p=0.99\right.$; Figure 1c), suggesting no deficit in single or simple sensory stimulation. The prepulse inhibition was however decreased in all four conditions and reached significance in two conditions $(78 \mathrm{~dB}, 120 \mathrm{~ms}$ prepulse-startle tone interval: $t_{112}=-2.17, p=0.03$; $86 \mathrm{~dB}, 120 \mathrm{~ms}$ prepulse - startle tone interval: $t_{112}=-2.10$, $p=0.04$; Figure 1c), suggesting a lack of inhibition of a subsequent tone, which may be indicative of hypersensitivity to subsequent or complex sensory stimuli.

Increased anxiety. Next, we examined anxiety levels in a standard elevated plus-maze. Treated $(n=52)$ animals spent $35 \%$ less time in the open arms $\left(t_{109}=-2.11\right.$, $p=0.04$; Figure 1c) and $14 \%$ more time in the closed arms $\left(67 \% \pm 3\right.$ for control; $77 \% \pm 3$ for treated; $t_{109}=2.46$, $p=0.01)$ than controls $(n=59)$, indicating increased anxiety in the VPA animals. This effect was not due to broad locomotor deficits as both total distances moved and average velocities, with which the animals moved through 
a

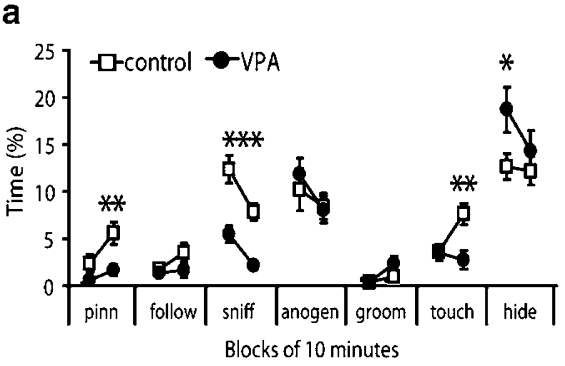

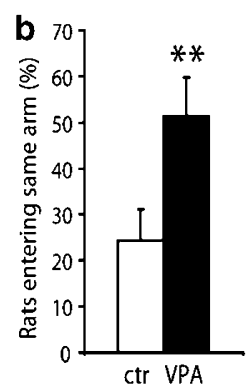
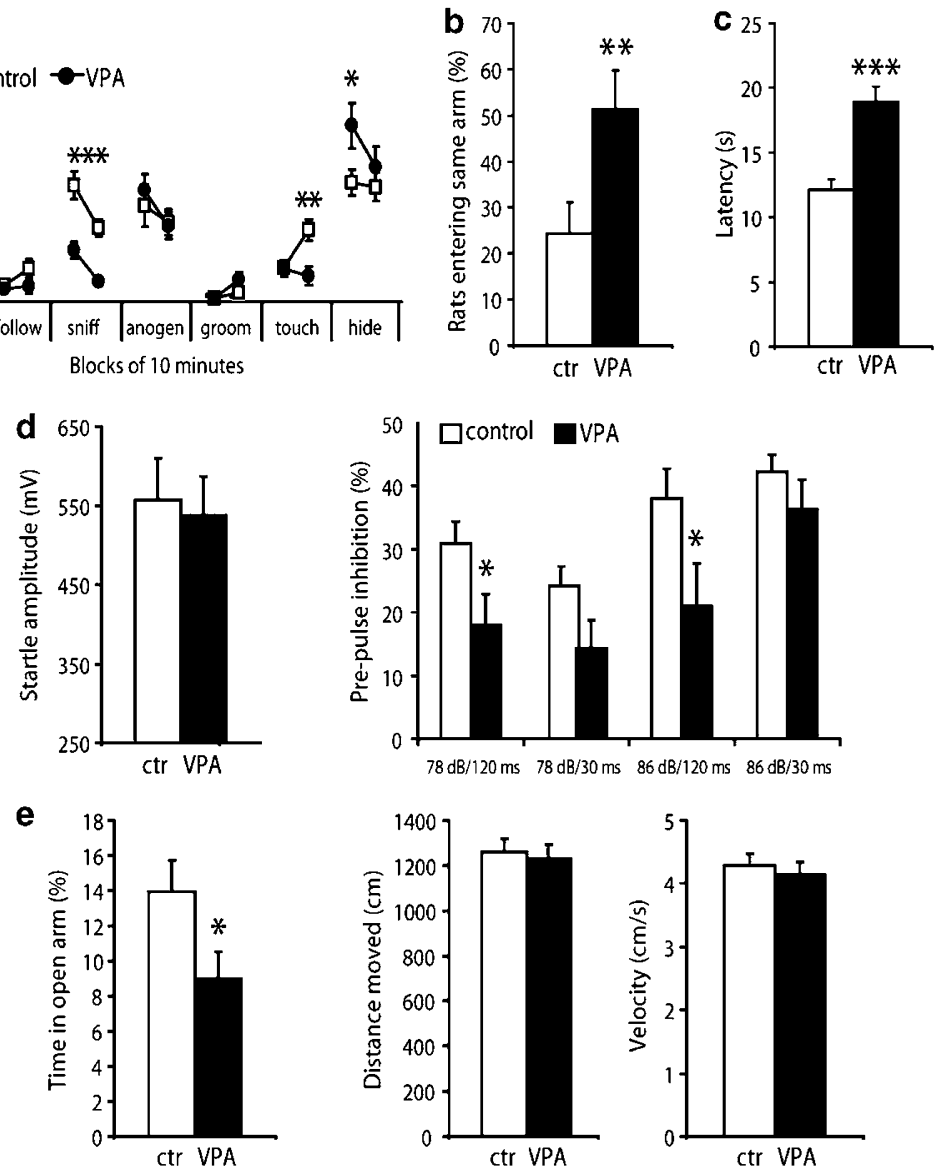

Figure I Behavioral characterization of the VPA model autism. Offspring of VPA-treated dams exhibit (a), impaired social interactions ( $n=10$ pairs/ group) (b), enhanced repetitive behavior in the Y-maze ( $n=43$ control; $n=35$ treated) (c), impaired thermal nociception in the hot plate test ( $n=36$ control; $n=39$ treated) (d), normal startle response to a tone, but impaired PPI ( $n=58$ control; $n=56$ treated) (e), increased anxiety in the EPM, but normal broad locomotion (distance moved and velocity) ( $n=59$ control; $n=52$ treated). Data show mean \pm SEM $(* p<0.05 ; * * p<0.0$ l; **** $p<0.00$ I)

the maze, were the same (distance: $t_{109}=-0.34, p=0.74$; velocity: $t_{109}=-0.56, p=0.58$; Figure $1 \mathrm{~d}$ ).

In summary, offspring of VPA-treated dams exhibited less social interactions, increased stereotypic, and repetitive tendencies, and less sensitivity to pain. Locomotion levels and reactions to simple auditory stimulation were normal in treated animals. However, more complex auditory stimulation in the PPI paradigm revealed impaired sensory habituation and/or response inhibition. Anxiety levels were increased in VPA-treated offspring, which indicated abnormal amygdala processing (Shekhar et al, 2005).

\section{Fear Processing}

Enhanced fear memories. During training, rats were exposed to three electric shocks ( $1 \mathrm{~mA}, 1 \mathrm{~s}$ every $60 \mathrm{~s}$ ) preceded by a tone $(20 \mathrm{~s}, 800 \mathrm{~Hz}, 80 \mathrm{~dB})$, which coterminated with the shock. Treated and control animals exhibited the same freezing levels in the shock period during training $\left(t_{150}=1.32, p=0.19 ; n=76\right.$ animals/group; Figure 2a), indicating normal fear acquisition and no differences in sensitivity to the shocks.

Fear memories were measured on two subsequent days starting 1, 30 and 90 days after fear conditioning for the context in which the fear was acquired, and the tone alone when presented in a novel context. Conditioned fear memory to both, tone and context, was highly elevated in VPA-treated animals at all time points measured, with the differences between groups becoming more striking with time, reaching up to $46 \%$ higher freezing after 3 months (tone: day $3, t_{150}=2.92, p=0.004 ;$ day $31, t_{140}=4.32$, $p<0.0000$; day 91, $t_{114}=4.56, p<0.0000$; context: day 2 , $t_{150}=4.00, p=0.0001$; day $30, t_{154}=4.86, p<0.0000$; day 90 , $t_{114}=3.92, p=0.0002 ; n=76$ animals/group; Figure $2 \mathrm{a}$ ).

Enhanced fear generalization. We also examined how these conditioned fear memories generalized to related stimuli. The fear response to a different tone $(400 \mathrm{~Hz}$, amplitude $1,80 \mathrm{~dB}$ ) or context (change of visual and odor cues) in treated animals was measured. We found that both groups expressed some fear when confronted with a different tone or a different context, but fear generalization was 24 and $45 \%$ greater in treated $(n=39)$ animals than controls $(n=36)$, respectively (tone: $t_{73}=3.49, p=0.0008$; context: $t_{73}=4.40, p<0.0000$; Figure $\left.2 \mathrm{~b}\right)$. We further computed the freezing ratio of the nonconditioned (NS, day 4) to the respective conditioned tone (day 3) and context stimuli (CS, day 2; NS/CS = generalization index $=\mathrm{GI}$ ). For both conditions, VPA-treated offspring exhibited a significantly higher generalization index (tone: 

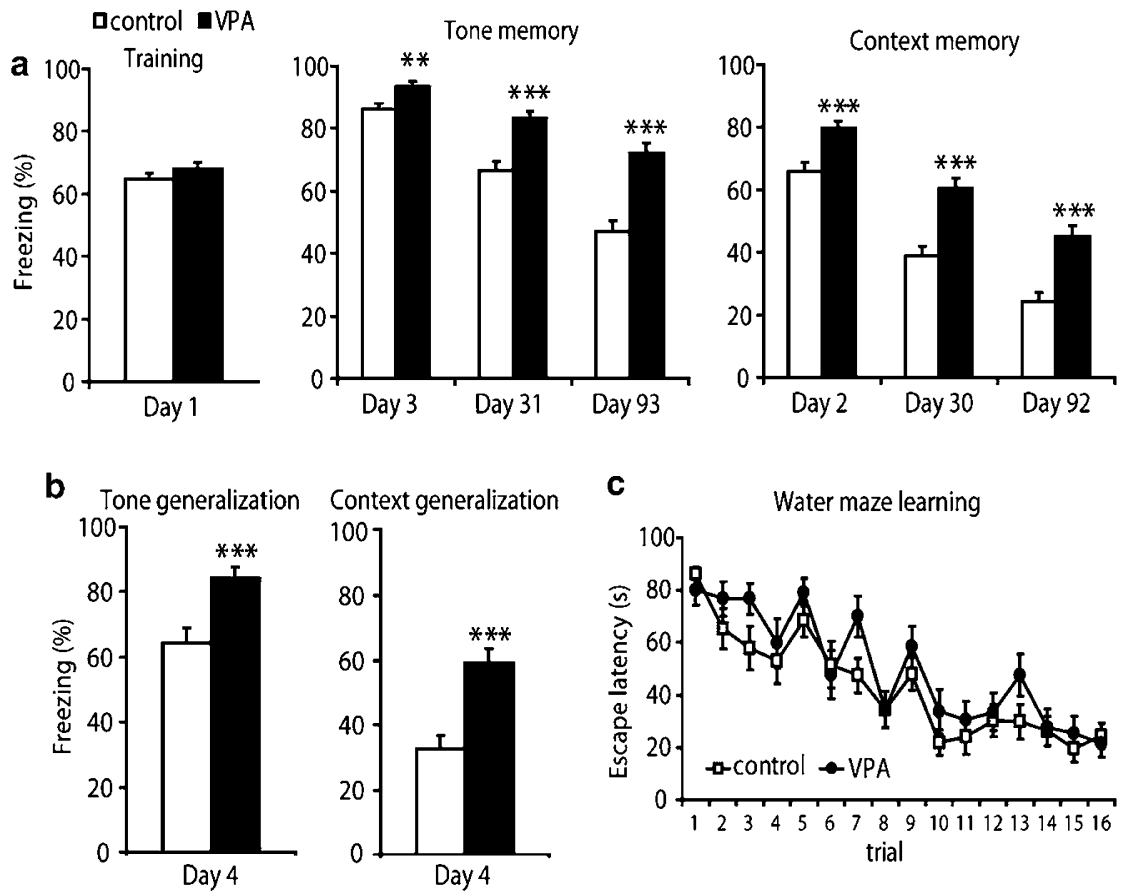

Figure 2 Enhanced fear memories and fear generalization. Offspring of VPA-treated dams exhibits (a), normal fear conditioning during training, but enhanced fear memories to the tone and context I, 30, and 90 days after training, with the differences becoming more pronounced with time ( $n=76$ pairs/ group) (b), enhanced fear generalization to a different tone and different context ( $n=36$ control; $n=39$ treated) (c), normal to slightly impaired spatial learning in the Morris Water Maze $(n=20$ control; $n=18$ treated). Data show mean \pm SEM (** $p<0.0$ I; $* * * p<0.00 I)$.

$\mathrm{GI}=0.68$ for control, $\mathrm{GI}=0.87$ for treated, $t_{73}=3.26$, $p=0.001$; context: $\mathrm{GI}=0.44$ for control, $\mathrm{GI}=0.68$ for treated, $\left.t_{73}=3.26, p=0.001\right)$. To address whether the enhanced fear generalization to the tone might be due to a ceiling effect in freezing, we further restricted the generalization analysis to those VPA-treated animals with the lowest freezing values (lower 50 and $30 \%$ of the VPA data set). We found that, even when excluding the highest freezing rats, the generalization index remained unchanged for the VPA-treated offspring and was still significantly different from the controls when considering the lower freezing $50 \%$ of VPA-treated rats $\left(\mathrm{GI}=0.86, t_{52}=2.33\right.$, $p=0.02)$ and in tendency also when considering the lower freezing $30 \%$ of the VPA-treated rats $\left(\mathrm{GI}=0.86, t_{45}=1.79\right.$, $p=0.08$ ), indicating that the enhanced generalization was not due to a ceiling effect. Thus, offspring of VPA-treated rats not only exhibited hyperfear memories, but also once conditioned overgeneralized fear to other NS.

Intact spatial learning and memory in the water maze. To check whether the increased memory associated with the amygdala was perhaps part of a general augmentation of memory mechanisms in the brain, we tested the rats on another memory task, the Morris water maze, which is highly dependent on the hippocampus (Morris et al, 1982), whereas it does not require the (basolateral) amygdala (Roozendaal et al, 1998). Rats were trained to find a submerged platform within four trials on each of four consecutive days. We found no differences between treated and control animals on any of the four training days or in a subsequent probe trial (all: $p>0.05, n=20$ for control and 18 for treated; Figure 2c). This may indicate that hippocampus-dependent memories are spared in VPAtreated animals, whereas amygdala-dependent fear memories are particularly affected in these animals.

Impaired fear extinction. Next, we asked whether the enhanced fear memories could be extinguished in VPAtreated rats as readily as in controls. For this purpose, subgroups of animals were re-conditioned and underwent several kinds of fear extinction trainings and an extinction index was computed (freezing post-extinction/freezing preextinction $\times 100)$. First, we applied contextual extinction training without any tone presentation. We found that in a post-extinction test, treated $(n=17)$ animals still exhibited $57 \%$ of the pre-extinction freezing levels, whereas control $(n=20)$ animals exhibited only $16 \%$ of the initial freezing levels, thus indicating a severe impairment of treated animals to extinguish the conditioning context $\left(t_{35}=2.24\right.$, $p=0.03$; Figure $3 a$ ). Second, we attempted to extinguish the fear to the tone, by presenting the tone repeatedly in the same context as during the conditioning. In this configuration, treated animals were again severely impaired in extinguishing fear responses to the tone in the postextinction test (control: $45 \pm 9 \%$ of pre-extinction freezing to tone; treated: $79 \pm 14 \%$ of pre-extinction freezing to tone; $t_{38}=2.14, p=0.04 ; n=20$ /group; Figure $3 \mathrm{~b}$ ). However, when we attempted to extinguish the tone in a novel context, the treated $(n=39)$ animals were able to unlearn fear responses to the tone almost as well as controls $(n=36)$ and exhibited only a tendency to impaired extinction (control: $43 \pm 4 \%$ of pre-extinction freezing to tone; treated: $53 \pm 4 \%$ of pre-extinction freezing to tone; $t_{73}=1.70$, $p=0.09$; Figure $3 c$ ). 

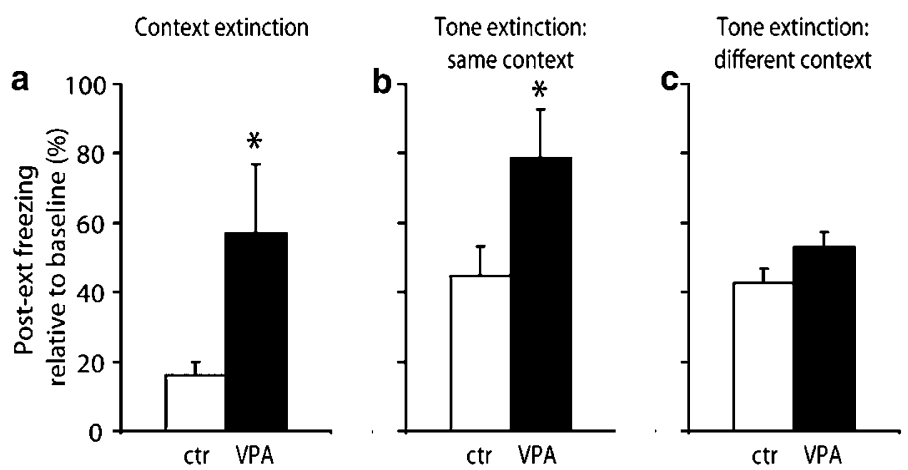

Figure 3 Impaired fear extinction. Depicted is the precentual decrease of post-extinction freezing from pre-extinction baseline levels. Offspring of VPAtreated dams exhibits impaired fear extinction to (a), the conditioning context ( $n=20$ control; $n=17$ treated) (b), a tone extinguished in the same context as during conditioning ( $n=20$ pairs/group), but almost normal extinction when (c), the tone is extinguished in a novel context ( $n=36$ control; $n=39$ treated). Data show mean \pm SEM $(* p<0.05)$.

In summary, amygdala-dependent fear memories were greatly enhanced, overgeneralized to other stimuli and more resistant to extinction in VPA-treated offspring, whereas performance in an amygdala-independent learning and memory task, spatial navigation in the water maze, remained normal.

\section{In Vitro Slice Electrophysiology in the Basolateral Amygdala}

To explore the possible cellular and synaptic changes in the amygdala of the VPA animals, we performed in vitro slice electrophysiological experiments on the LA, the sensory relay station between the aversive shock and the conditioned tone stimulus (LeDoux et al, 1990), and examined how pyramidal neurons (Figure 4a) responded to stimulation of the amygdala and how synaptic connections reacted to a Hebbian pairing protocol.

Increased reactivity to stimulation. We found that strong stimulation using MEA can activate the amygdala circuit to produce reverberant activity that persists for seconds without any further stimulation (Figure $4 \mathrm{~b}$ ). Therefore, we compared these evoked activity states in control and VPAtreated animals. For the equivalent stimulation, nearly double the fraction of cells (83\%) from VPA-treated animals displayed this reverberant activity compared to $47 \%$ of cells from control animals (control: 10 out of 21 cells; treated: 15 out of 18 cells, $\chi_{1}^{2}=5.37, p=0.02$; Figure $4 c$ ). Furthermore, among the cells displaying reverberant activity states, the frequency of activity was significantly higher in treated rats $\left(t_{23}=-2.39, p=0.02 ; n=15\right.$; Figure $\left.4 c\right)$.

Increased long-term potentiation. As synaptic plasticity in the LA is thought to underlie fear memories (Quirk et al, 1995; Collins and Pare, 2000; Repa et al, 2001; Bauer et al, 2002), we also tested for LTP in VPA-treated rats. We found that the potentiation caused by a classical pairing protocol with an extracellular electrode placed just medial to the LA was significantly increased in treated rats $\left(t_{12}=3.12\right.$ $p=0.009 ; n=15$ for control, 10 for treated; Figure $4 c$ ).
Impaired inhibition. We finally examined the inhibitory inputs into whole-cell patched principal neurons clamped at a voltage equal to the reversal potential for AMPA receptors $\left(V_{\text {hold }}=+10 \mathrm{mV}\right)$, following MEA extracellular stimulation with increasing amplitude. We measured the total inhibitory current generated by a progressively greater stimulus and compared the slices from VPA-treated animals with those from the controls. The resulting stimulus-response curve (Figure $4 \mathrm{~d}$ ) indicated that inhibition in the LA was highly significantly decreased in VPA-treated rats as compared to control rats $\left(\mathrm{F}_{1,42}=10.50, p=0.002\right.$, Figure $\left.4 \mathrm{~d}\right)$.

Due to excessive myelin formation with increasing age, these experiments could not be performed at the same age as the behavioral experiments, thus it cannot be entirely excluded that that these cellular and synaptic effects might recover with age.

In summary, the LA network of VPA-treated animals was hyperreactive to stimulation and hyperplastic to intense synaptic stimulation. Moreover, inhibition was impaired in the LA.

\section{DISCUSSION}

In the present study, we show that a single injection of VPA to pregnant rats on gestational day 12.5 yielded offspring with a behavioral pattern strikingly similar to that observed in autism. VPA-treated rat offspring exhibited symptoms such as decreased social interactions, increased repetitive behaviors, and less sensitivity to pain-some of the hallmark features of autism. Locomotion levels and reactions to simple auditory stimulation were normal in treated animals, suggesting no motor or simple sensory impairments. However, more complex auditory stimulation in the prepulse inhibition paradigm revealed impaired sensorimotor gating, which may be interpreted as impaired sensory habituation and/or impaired response inhibition to complex sensory input, and may lead to sensory overload and the strong reactions to sensory stimulation, commonly observed in autism. These results show that the VPA rat model of autism is reproducible, as it is largely in accordance with previous behavioral data (Schneider and Przewlocki, 2005). 

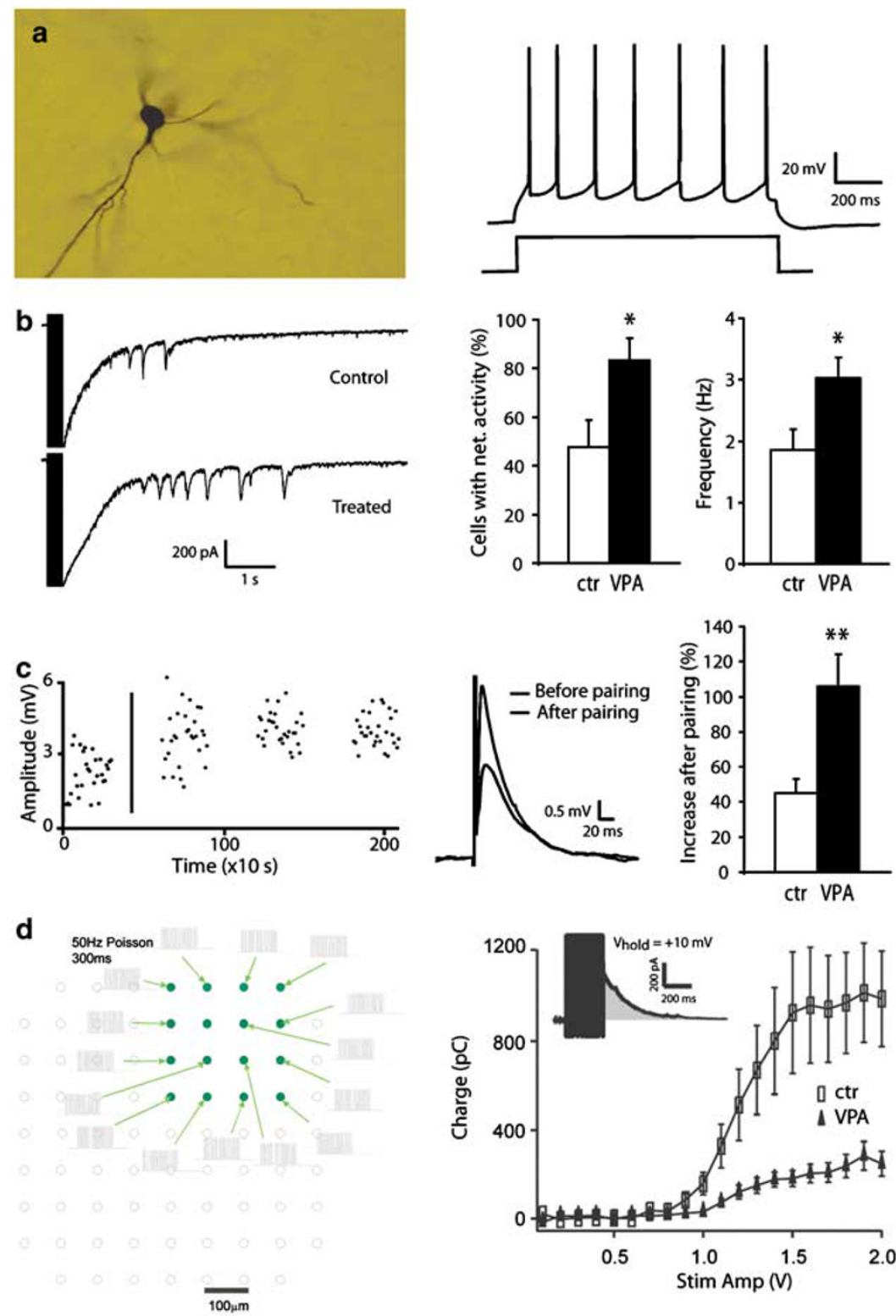

Figure 4 Increased network excitability, enhanced LTP and impaired inhibition in the LA. (a) Example of a principal cell of the LA and corresponding firing pattern. (b) Response to multi-electrode array (MEA) network stimulation in a voltage-clamped ( $-80 \mathrm{mV}$ ) pyramidal cell, illustrating the typical network activity observed with this stimulation in control and VPA-treated offspring. The percentage of cells showing persistent reverberant activity is higher in treated ( 15 out of 18 cells) than in control rats ( 10 out of 21 cells). Among the cells showing this activity, the frequency of reverberant events is higher in treated than in control rats. (c) LTP is enhanced in treated rats. The left graph displays an example of the response amplitudes to extracellular stimulation before and after the pairing protocol (represented by the gray bar), and the middle graph depicts mean amplitudes before and after pairing. The bar graph represents the percentage increase in response amplitude after the pairing for control $(n=15)$ and treated $(n=10)$ rats. (d) The left graph shows a scheme of MEA stimulation with 16 electrodes $(50 \mathrm{~Hz}$ Poisson train, $300 \mathrm{~ms})$ in principal cells of the LA. The right graphs display the inhibitory response curve of principal cells voltage clamped at $+10 \mathrm{mV}$ to MEA stimulation with increasing amplitude. In the upper left corner, the typical response of a single cell is shown. The total area below the curve (charge) is measured for a series of different stimulation intensities. The inhibitory charge generated for all intensities is significantly smaller in the VPA-treated animals. Data show mean \pm SEM $(* p<0.05$; ** $p<0.0$ I).

This study further provides the first clues that abnormal anxiety and fear processing are significant behavioral features of this animal model and could be a major factor shaping behavior in autism. Fear memories were not only stronger and longer lasting in VPA-treated offspring, but generalized more to novel stimuli and were harder to extinguish.

The study also shows for the first time exaggerated cellular responses to electrical stimulation, enhanced synaptic plasticity, and a deficit in inhibition in the amygdala. The hyperreactivity and hyperplasticity could be due to the impaired inhibition in the treated rats.

We suggest that enhanced fear processing and memories could perhaps underlie what has been considered to be core symptoms of autism, such as impaired social interactions and resistance to rehabilitation. These data also support the view that enhanced, rather than weak amygdala function, may be an important part of the pathology in autism. 


\section{The VPA Rat Model of Autism - a Valid Model?}

Animal models of disorders identified in humans, especially disorders of high-level cognitive functions, are likely to suffer from many shortcomings. Results from animal models must therefore be interpreted with caution and can only provide suggested new directions to facilitate research of the disorder in humans. As an animal model of autism, the VPA model seems to be the closest possible insult-based model that allows a systematic study from the synaptic to the behavioral level. Several case studies (Christianson et al, 1994; Williams and Hersh, 1997; Williams et al, 2001), as well as a population study (Moore et al, 2000), reported a link between the administration of the anticonvulsant drug VPA during pregnancy and the risk of giving birth to an autistic child. Although several rodent studies explored and established the teratogenic effects of prolonged VPA administration during pregnancy in general (Vorhees 1987a,b) and in the context of autism (Narita et al, 2002; Wagner et al, 2006), we chose the VPA rat model of autism proposed by Rodier et al (1996). This model focuses specifically at the time of neural tube closure on gestational day 12.5 , which is believed to be a particularly vulnerable time point for insults that may produce autistic symptoms. Indeed, striking similarities were observed between such VPA-treated rat offspring and autism, including: (i) a reduced number of motor cells in cranial nerve motor nuclei in the brain stem (Rodier et al, 1996), (ii) a reduced number of Purkinje cells in the cerebellum, observed both in VPA-treated offspring (Rodier et al, 1997; Ingram et al, 2000) and autism (Ritvo et al, 1986; Kemper and Bauman, 1998), (iii) decreased social interactions, increased repetitive behaviors, lower sensitivity to pain, impaired sensorimotor gating and increased anxiety described in VPA-treated offspring (Schneider and Przewlocki, 2005; Schneider et al, 2006) and commonly found in the autistic spectrum (American Psychiatric Association, 1994; Muris et al, 1998; McAlonan et al, 2002; Perry et al, 2007).

Our results confirm many of the previous behavioral findings on this particular VPA rat model of autism (Schneider and Przewlocki, 2005; Schneider et al, 2006) and show that this animal model is indeed reproducible. Altogether, this cumulative evidence obtained with the VPA rat model of autism indicates remarkable similarities with the human autistic phenotype.

\section{A Novel Role Suggested for the Amygdala in Autism}

Both microscopic and macroscopic studies reveal alterations in the morphology of the amygdala in autism (Kemper and Bauman, 1998; Aylward et al, 1999; Sparks et al, 2002; Schumann et al, 2004; Schumann and Amaral, 2006), indicating a dysfunction in this region that might possibly contribute to the autistic pathology. The current 'amygdala theory of autism' is based on the lost capability to evaluate socioemotional information and lost drive for social interaction derived from lesioning studies in monkeys and a few functional imaging studies in humans (Baron-Cohen et al, 2000; Sweeten et al, 2002; Pelphrey et al, 2004; Bachevalier and Loveland, 2006; Schultz, 2005), which implies that a hypofunctioning amygdala underlies autism.
The amygdala is however also a crucial brain structure implicated in mediating innate and acquired fear memories as well as generalized anxiety (Davis and Whalen, 2001; LeDoux, 2003). Even though the hypothesis that the amygdala might underlie abnormal fear processing in autism has been brought forward recently (Amaral et al, 2003; Schumann and Amaral, 2006), the present study is the first to show that fear memories are greatly enhanced, overly generalized to novel stimuli, and harder to extinguish in an animal model of autism. These results are in fact also consistent with some less cited autistic features such as enhanced fears and phobias originally described by Kanner (1943) and reported in some more recent studies (Muris et al, 1998; Gillott et al, 2001; Evans et al, 2005). It is however not clear if enhanced fears are common across the autistic spectrum as some high-functioning autists do not seem to display abnormal conditioned fear (Bernier et al, 2005).

We also found that behavioral alterations that could be due to abnormal amygdala processing are indeed accompanied by direct physiological alterations in the lateral complex of the amygdala. In particular, inhibition is lowered and the circuit is hyperreactive when electrically stimulated. Synaptic plasticity is also boosted suggesting that the amygdala turned hyperplastic following a single exposure of the embryo to VPA. Considering that LTP in the basolateral amygdala is thought to be the key cellular mechanism underlying fear memory formation (Quirk et al, 1995; Rogan et al, 1997; Collins and Pare, 2000; Repa et al, 2001; Bauer et al, 2002), hyperreactivity and hyperplasticity are strong candidates mediating the enhanced and overgeneralized fear memories in the VPA-treated rats.

The amygdala also possesses a powerful inhibitory interneuron system (Takagi and Yamamoto, 1981; Washburn and Moises, 1992a,b), which tightly controls neuronal activity and plasticity (Li et al, 1996; Szinyei et al, 2000; Bissiere et al, 2003; Shaban et al, 2006). In fact, amygdalar disinhibition induced either by GABAergic blockade (Isoardi et al, 2004), genetic knockout of the

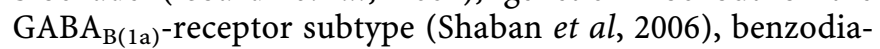
zepine withdrawal (Isoardi et al, 2004), dopamine receptor activation (Bissiere et al, 2003), or stress (Rodriguez Manzanares et al, 2005), can result in amygdaloid hyperexcitability (Isoardi et al, 2004; Rodriguez Manzanares et al, 2005) and facilitate LTP induction (Bissiere et al, 2003; Rodriguez Manzanares et al, 2005; Shaban et al, 2006), which have been associated not only with enhanced fear memories (Isoardi et al, 2004; Rodriguez Manzanares et al, 2005), but also with overgeneralization of conditioned fear to neutral stimuli (Shaban et al, 2006). These reports suggest that the hyperreactivity and hyperplasticity observed in this study may be due to a deficit in inhibition in the amygdala. Indeed, we found a greatly reduced level of inhibition during parallel electrical stimulation of the amygdala.

\section{Indirect Effects of Non-Amygdala Regions}

The extinction of conditioned fear involves the amygdala (Falls et al, 1992; Lu et al, 2001; Barad, 2006), as well as a loop between the amygdala and the medial prefrontal cortex (Sotres-Bayon et al, 2004, 2006). The prefrontal cortex gains 
increasing importance during the extinction process by inhibiting amygdaloid responses to fear conditioned stimuli (Rosenkranz et al, 2003), presumably through excitatory projections on inhibitory neurons in the amygdala (Quirk et al, 2003). A deficit in the inhibitory system in the amygdala could therefore also compromise the effectiveness of the projections from the prefrontal cortex in mediating extinction. An alternative explanation is a deficit in the medial prefrontal cortex itself. Indeed, the prefrontal cortex was suggested to be crucially affected in autism (Bachevalier and Loveland, 2006). A recent study however indicates that the medial prefrontal cortex is also hyperreactive and hyperplastic in VPA-treated offspring (TR \& HM, unpublished data), suggesting that the failure in extinction may be more related to a loss of inhibition in the amygdala.

Contextual fear conditioning is dependent not only on the amygdala, but also on the hippocampus (Phillips and LeDoux, 1992), suggesting that the enhancement of CFC found in the present study may be due to hyperfunctioning of the hippocampus. Spatial learning and memory performance using the Morris water maze - a task which depends on the hippocampus (Morris et al, 1982) - were however not affected, further implicating hyperfunctioning of the amygdaloid component in the fear conditioning data. Further experiments are however required to determine whether other forms of memories, such as reward-based, rather than punishment-based, conditioning, are affected in this animal model. Recent studies in our lab also indicate that synaptic plasticity is greatly enhanced in the neocortex of these VPA rats (Rinaldi et al, in press), thus it is possible that memory tasks, which depend more on the neocortex, are also enhanced. Further studies to test this hypothesis are in progress.

\section{Abnormal Amygdala Functioning and Fear Processing - Core Symptom of Autism?}

Hirstein et al (2001) suggested that an excessively functioning amygdala may account for the increased autonomic responses in autistic children (Hirstein et al, 2001; Corbett et al, 2006) and the consequent enhanced stress may lead to their withdrawal from an overstimulating world. The present study suggests that enhanced anxiety and fear processing could perhaps further amplify an aversion to complex environmental stimulation typical of social conditions, leading to impaired social interactions and resistance to rehabilitation. The presented data also supports the view that enhanced, rather than weak amygdala function may be part of the pathology in autism. In accordance with these hypotheses are the findings that a hyperreactive amygdala has been associated with social phobia (Stein et al, 2002; Tillfors, 2004), whereas enhanced sociability as encountered in Williams syndrome, is associated with a hyporesponsive amygdala (Meyer-Lindenberg et al, 2005). A recent study screening for autism-like symptoms in children with mood and anxiety disorders also found that up to $62 \%$ of these children fall into the autistic spectrum and might qualify for a possible diagnosis of autism (Towbin et al, 2005). Thus, we would like to speculate that enhanced amygdaloid processing in the basolateral complex, possibly due to a failure in inhibition, may account for enhanced fears (Isoardi et al, 2004;
Rodriguez Manzanares et al, 2005) and lead to a reduction in social interactions. This is supported by a rodent study showing that lesioning the BLA, the main relay station for fear associations, impaired not only fear conditioning - a well established finding (LeDoux et al, 1990) — but also led to an increase, rather than a decrease, in social interactions (Woolley et al, 2006). Thus, it is conceivable that an overly active and plastic amygdala may produce enhanced anxiety and fear processing, leading to social withdrawal to avoid aversive, fear-evoking exposure.

\section{Conclusion}

This study provides the first experimental demonstration of enhanced, overgeneralized and extinction-resistant conditioned fear memories in a rat model of autism. These behavioral symptoms may be caused by the hyperreactivity and hyperplasticity discovered in the LA of VPA-treated offspring, which may be due to impairments in the inhibitory system of the amygdala. Therefore, we hypothesize that enhanced anxiety and fear processing in the amygdala may contribute to an 'aversive world' syndrome in autism, leading to withdrawal from social interactions and resistance to rehabilitation. It is however important to caution that this is only an animal model of autism and that considerable amount of work still has to be performed to determine the relevance of these findings to the human condition.

\section{DISCLOSURE/CONFLICT OF INTEREST}

The authors declare that, except for income received from the primary employer, no financial support or compensation has been received from any individual or corporate entity over the past 3 years for research or professional service and there are no personal financial holdings that could be perceived as constituting a potential conflict of interest.

\section{REFERENCES}

Adolphs R, Sears L, Piven J (2001). Abnormal processing of social information from faces in autism. J Cogn Neurosci 13: 232-240.

Amaral DG, Bauman MD, Schumann CM (2003). The amygdala and autism: implications from non-human primate studies. Genes Brain Behav 2: 295-302.

Arndt TL, Stodgell CJ, Rodier PM (2005). The teratology of autism. Int J Dev Neurosci 23: 189-199.

American Psychiatric Association (1994). Diagnostic and Statistical Manual of Mental Disorders, 4th edn. Washington, DC.

Aylward EH, Minshew NJ, Goldstein G, Honeycutt NA, Augustine AM, Yates KO et al (1999). MRI volumes of amygdala and hippocampus in non-mentally retarded autistic adolescents and adults. Neurology 53: 2145-2150.

Bachevalier J (1994). Medial temporal lobe structures and autism: a review of clinical and experimental findings. Neuropsychologia 32: 627-648.

Bachevalier J, Loveland KA (2006). The orbitofrontal-amygdala circuit and self-regulation of social-emotional behavior in autism. Neurosci Biobehav Rev 30: 97-117.

Barad M (2006). Is extinction of fear erasure or inhibition? Why both, of course. Learn Mem 13: 108-109. 
Baron-Cohen S, Ring HA, Bullmore ET, Wheelwright S, Ashwin C, Williams SC (2000). The amygdala theory of autism. Neurosci Biobehav Rev 24: 355-364.

Baron-Cohen S, Ring HA, Wheelwright S, Bullmore ET, Brammer MJ, Simmons A et al (1999). Social intelligence in the normal and autistic brain: an fMRI study. Eur J Neurosci 11: 1891-1898.

Bauer EP, Schafe GE, LeDoux JE (2002). NMDA receptors and L-type voltage-gated calcium channels contribute to long-term potentiation and different components of fear memory formation in the lateral amygdala. J Neurosci 22: 5239-5249.

Bernier R, Dawson G, Panagiotides H, Webb S (2005). Individuals with autism spectrum disorder show normal responses to a fear potential startle paradigm. J Autism Dev Disord 35: 575-583.

Bissiere S, Humeau Y, Luthi A (2003). Dopamine gates LTP induction in lateral amygdala by suppressing feedforward inhibition. Nat Neurosci 6: 587-592.

Christianson AL, Chesler N, Kromberg JG (1994). Fetal valproate syndrome: clinical and neuro-developmental features in two sibling pairs. Dev Med Child Neurol 36: 361-369.

Collins DR, Pare D (2000). Differential fear conditioning induces reciprocal changes in the sensory responses of lateral amygdala neurons to the CS(+) and CS(-). Learn Mem 7: 97-103.

Corbett BA, Mendoza S, Abdullah M, Wegelin JA, Levine S (2006). Cortisol circadian rhythms and response to stress in children with autism. Psychoneuroendocrinology 31: 59-68.

Critchley H, Daly E, Phillips M, Brammer M, Bullmore E, Williams $S$ et al (2000). Explicit and implicit neural mechanisms for processing of social information from facial expressions: a functional magnetic resonance imaging study. Hum Brain Mapp 9: 93-105.

Davis M, Whalen PJ (2001). The amygdala: vigilance and emotion. Mol Psychiatry 6: 13-34.

Evans DW, Canavera K, Kleinpeter FL, Maccubbin E, Taga K (2005). The fears, phobias and anxieties of children with autism spectrum disorders and down syndrome: comparisons with developmentally and chronologically age matched children. Child Psychiatry Hum Dev 36: 3-26.

Falls WA, Miserendino MJ, Davis M (1992). Extinction of fearpotentiated startle: blockade by infusion of an NMDA antagonist into the amygdala. J Neurosci 12: 854-863.

Gillott A, Furniss F, Walter A (2001). Anxiety in high-functioning children with autism. Autism 5: 277-286.

Hirstein W, Iversen P, Ramachandran VS (2001). Autonomic responses of autistic children to people and objects. Proc Biol Sci 268: $1883-1888$.

Ingram JL, Peckham SM, Tisdale B, Rodier PM (2000). Prenatal exposure of rats to valproic acid reproduces the cerebellar anomalies associated with autism. Neurotoxicol Teratol 22: 319-324.

Isoardi NA, Martijena ID, Carrer HF, Molina VA (2004). Increased fear learning coincides with neuronal dysinhibition and facilitated LTP in the basolateral amygdala following benzodiazepine withdrawal in rats. Neuropsychopharmacology 29: $1852-1864$.

Kahne D, Tudorica A, Borella A, Shapiro L, Johnstone F, Huang W et al (2002). Behavioral and magnetic resonance spectroscopic studies in the rat hyperserotonemic model of autism. Physiol Behav 75: 403-410.

Kanner L (1943). Autistic disturbances of affective contact. Nerv Child 2: 217-250.

Kemper TL, Bauman M (1998). Neuropathology of infantile autism. J Neuropathol Exp Neurol 57: 645-652.

Kling A, Brothers L (1992). The Amygdala and Social Behavior. Neurobiological Aspects of Emotion, Memory, and Mental Dysfunction. J. Aggleton, Wiley: New York.

La Mendola D, Markram K, Rinaldi T, Sandi C, Markram H (2006). Hyper-fear, reduced fear extinction and weakened inhibition in the amygdala in an animal model of autism. FENS (Abstract, 3, A026.12).
LeDoux J (2003). The emotional brain, fear, and the amygdala. Cell Mol Neurobiol 23: 727-738.

LeDoux JE, Cicchetti P, Xagoraris A, Romanski LM (1990). The lateral amygdaloid nucleus: sensory interface of the amygdala in fear conditioning. J Neurosci 10: 1062-1069.

Li XF, Armony JL, LeDoux JE (1996). GABAA and GABAB receptors differentially regulate synaptic transmission in the auditory thalamo-amygdala pathway: an in vivo microiontophoretic study and a model. Synapse 24: 115-124.

Lu KT, Walker DL, Davis M (2001). Mitogen-activated protein kinase cascade in the basolateral nucleus of amygdala is involved in extinction of fear-potentiated startle. J Neurosci 21: RC162.

Markram K, La Mendola D, Rinaldi T, Sandi C, Markram H (2005). Enhanced fear and reduced fear extinction in an animal model of autism. Soc Neurosci (Abstract, 448.3).

McAlonan GM, Daly E, Kumari V, Critchley HD, van Amelsvoort $\mathrm{T}$, Suckling J et al (2002). Brain anatomy and sensorimotor gating in Asperger's syndrome. Brain 125: 1594-1606.

Meyer-Lindenberg A, Hariri AR, Munoz KE, Mervis CB, Mattay VS, Morris CA et al (2005). Neural correlates of genetically abnormal social cognition in Williams syndrome. Nat Neurosci 8: 991-993.

Moore SJ, Turnpenny P, Quinn A, Glover S, Lloyd DJ, Montgomery $\mathrm{T}$ et al (2000). A clinical study of 57 children with fetal anticonvulsant syndromes. J Med Genet 37: 489-497.

Morris RG, Garrud P, Rawlins JN, O'Keefe J (1982). Place navigation impaired in rats with hippocampal lesions. Nature 297: 681-683.

Muris P, Steerneman P, Merckelbach H, Holdrinet I, Meesters C (1998). Comorbid anxiety symptoms in children with pervasive developmental disorders. J Anxiety Disord 12: 387-393.

Narita N, Kato M, Tazoe M, Miyazaki K, Narita M, Okado N (2002). Increased monoamine concentration in the brain and blood of fetal thalidomide- and valproic acid-exposed rat: putative animal models for autism. Pediatr Res 52: 576-579.

Newman JD, Bachevalier J (1997). Neonatal ablations of the amygdala and inferior temporal cortex alter the vocal response to social separation in rhesus macaques. Brain Res 758: $180-186$.

Pelphrey K, Adolphs R, Morris JP (2004). Neuroanatomical substrates of social cognition dysfunction in autism. Ment Retard Dev Disabil Res Rev 10: 259-271.

Perry W, Minassian A, Lopez B, Maron L, Lincoln A (2007). Sensorimotor gating deficits in adults with autism. Biol Psychiatry 61: 482-486.

Phillips RG, LeDoux JE (1992). Differential contribution of amygdala and hippocampus to cued and contextual fear conditioning. Behav Neurosci 106: 274-285.

Pierce K, Muller RA, Ambrose J, Allen G, Courchesne E (2001). Face processing occurs outside the fusiform 'face area' in autism: evidence from functional MRI. Brain 124: 2059-2073.

Quirk GJ, Likhtik E, Pelletier JG, Pare D (2003). Stimulation of medial prefrontal cortex decreases the responsiveness of central amygdala output neurons. J Neurosci 23: 8800-8807.

Quirk GJ, Repa C, LeDoux JE (1995). Fear conditioning enhances short-latency auditory responses of lateral amygdala neurons: parallel recordings in the freely behaving rat. Neuron 15: 1029-1039.

Rasalam AD, Hailey H, Williams JH, Moore SJ, Turnpenny PD, Lloyd DJ et al (2005). Characteristics of fetal anticonvulsant syndrome associated autistic disorder. Dev Med Child Neurol 47: 551-555.

Repa JC, Muller J, Apergis J, Desrochers TM, Zhou Y, LeDoux JE (2001). Two different lateral amygdala cell populations contribute to the initiation and storage of memory. Nat Neurosci 4: 724-731.

Rinaldi T, Kulangara K, Antoniello K, Markram H (submitted). Elevated NMDA receptor levels and enhanced postsynaptic long 
term potentiation induced by prental exposure to valproic acid. Proc Natl Acad Sci USA (in press).

Ritvo ER, Freeman BJ, Scheibel AB, Duong T, Robinson H, Guthrie $\mathrm{D}$ et al (1986). Lower Purkinje cell counts in the cerebella of four autistic subjects: initial findings of the UCLA-NSAC Autopsy Research Report. Am J Psychiatry 143: 862-866.

Rodier PM, Ingram JL, Tisdale B, Croog VJ (1997). Linking etiologies in humans and animal models: studies of autism. Reprod Toxicol 11: 417-422.

Rodier PM, Ingram JL, Tisdale B, Nelson S, Romano J (1996). Embryological origin for autism: developmental anomalies of the cranial nerve motor nuclei. J Comp Neurol 370: 247-261.

Rodriguez Manzanares PA, Isoardi NA, Carrer HF, Molina VA (2005). Previous stress facilitates fear memory, attenuates GABAergic inhibition, and increases synaptic plasticity in the rat basolateral amygdala. J Neurosci 25: 8725-8734.

Rogan MT, Staubli UV, LeDoux JE (1997). Fear conditioning induces associative long-term potentiation in the amygdala. Nature 390: 604-607.

Roozendaal B, Sapolsky RM, McGaugh JL (1998). Basolateral amygdala lesions block the disruptive effects of long-term adrenalectomy on spatial memory. Neuroscience 84: 453-465.

Rosenkranz JA, Moore H, Grace AA (2003). The prefrontal cortex regulates lateral amygdala neuronal plasticity and responses to previously conditioned stimuli. J Neurosci 23: 11054-11064.

Schneider T, Przewlocki R (2005). Behavioral alterations in rats prenatally exposed to valproic acid: animal model of autism. Neuropsychopharmacology 30: 80-89.

Schneider T, Turczak J, Przewlocki R (2006). Environmental enrichment reverses behavioral alterations in rats prenatally exposed to valproic Acid: issues for a therapeutic approach in autism. Neuropsychopharmacology 31: 36-46.

Schultz RT (2005). Developmental deficits in social perception in autism: the role of the amygdala and fusiform face area. Int J Dev Neurosci 23: 125-141.

Schumann CM, Amaral DG (2006). Stereological analysis of amygdala neuron number in autism. J Neurosci 26: 7674-7679.

Schumann CM, Hamstra J, Goodlin-Jones BL, Lotspeich LJ, Kwon $\mathrm{H}$, Buonocore $\mathrm{MH}$ et al (2004). The amygdala is enlarged in children but not adolescents with autism; the hippocampus is enlarged at all ages. J Neurosci 24: 6392-6401.

Shaban H, Humeau Y, Herry C, Cassasus G, Shigemoto R, Ciocchi $S$ et al (2006). Generalization of amygdala LTP and conditioned fear in the absence of presynaptic inhibition. Nat Neurosci 9: $1028-1035$.

Shekhar A, Truitt W, Rainnie D, Sajdyk T (2005). Role of stress, corticotrophin releasing factor (CRF) and amygdala plasticity in chronic anxiety. Stress 8: 209-219.

Sotres-Bayon F, Bush DE, LeDoux JE (2004). Emotional perseveration: an update on prefrontal-amygdala interactions in fear extinction. Learn Mem 11: 525-535.
Sotres-Bayon F, Cain CK, Ledoux JE (2006). Brain mechanisms of fear extinction: historical perspectives on the contribution of prefrontal cortex. Biol Psychiatry 60: 329-336.

Sparks BF, Friedman SD, Shaw DW, Aylward EH, Echelard D, Artru AA et al (2002). Brain structural abnormalities in young children with autism spectrum disorder. Neurology 59: 184-192.

Stein MB, Goldin PR, Sareen J, Zorrilla LT, Brown GG (2002). Increased amygdala activation to angry and contemptuous faces in generalized social phobia. Arch Gen Psychiatry 59: 1027-1034.

Sweeten TL, Posey DJ, Shekhar A, McDougle CJ (2002). The amygdala and related structures in the pathophysiology of autism. Pharmacol Biochem Behav 71: 449-455.

Szinyei C, Heinbockel T, Montagne J, Pape HC (2000). Putative cortical and thalamic inputs elicit convergent excitation in a population of GABAergic interneurons of the lateral amygdala. J Neurosci 20: 8909-8915.

Takagi M, Yamamoto C (1981). The long-lasting inhibition recorded in vitro from the lateral nucleus of the amygdala. Brain Res 206: 474-478.

Tillfors M (2004). Why do some individuals develop social phobia? A review with emphasis on the neurobiological influences. Nord J Psychiatry 58: 267-276.

Towbin KE, Pradella A, Gorrindo T, Pine DS, Leibenluft E (2005). Autism spectrum traits in children with mood and anxiety disorders. J Child Adolesc Psychopharmacol 15: 452-464.

Vorhees CV (1987a). Behavioral teratogenicity of valproic acid: selective effects on behavior after prenatal exposure to rats. Psychopharmacology (Berl) 92: 173-179.

Vorhees CV (1987b). Teratogenicity and developmental toxicity of valproic acid in rats. Teratology 35: 195-202.

Wagner GC, Reuhl KR, Cheh M, McRae P, Halladay AK (2006). A new neurobehavioral model of autism in mice: pre- and postnatal exposure to sodium valproate. J Autism Dev Disord 36: 779-793.

Washburn MS, Moises HC (1992a). Electrophysiological and morphological properties of rat basolateral amygdaloid neurons in vitro. J Neurosci 12: 4066-4079.

Washburn MS, Moises HC (1992b). Inhibitory responses of rat basolateral amygdaloid neurons recorded in vitro. Neuroscience 50: $811-830$.

Williams G, King J, Cunningham M, Stephan M, Kerr B, Hersh JH (2001). Fetal valproate syndrome and autism: additional evidence of an association. Dev Med Child Neurol 43: 202-206.

Williams PG, Hersh JH (1997). A male with fetal valproate syndrome and autism. Dev Med Child Neurol 39: 632-634.

Woolley ML, Haman M, Higgins GA, Ballard TM (2006). Investigating the effect of bilateral amygdala lesions on fear conditioning and social interaction in the male Mongolian gerbil. Brain Res 1078: 151-158.

Yadin E, Friedman E, Bridger WH (1991). Spontaneous alternation behavior: an animal model for obsessive-compulsive disorder? Pharmacol Biochem Behav 40: 311-315. 